\title{
Psychometric properties of the Perceived Stress Scale (PSS): Measurement invariance between athletes and non-athletes and construct validity
}

\author{
Yi-Hsiang Chiu ${ }^{1}$, Frank Jing-Horng Lu ${ }^{\text {Corresp.. }}{ }^{2}$, Ju-Han Lin ${ }^{3}$, Chiao-Lin Nien ${ }^{4}$, Ya-Wen Hsu ${ }^{5}$, Hong-Yu Liu \\ ${ }^{1}$ Graduate Institute of Physical Education, National Taiwan Sport University, Taoyuan, Taiwan, Taiwan \\ 2 Graduate Institute of Sport Coaching Science, Chinese Culture University, Taipei, Taiwan, Taiwan \\ 3 Department of Physical Education and Kinesiology, National Dong Hwa University, Hua Lien, Taiwan, Taiwan \\ 4 Department of Physical Education, National Taiwan University of Sport, Taichung, Taiwan \\ 5 Department of Physical Education, Health, and Recreation, National Chia-Yi University, Chia-Yi, Taiwan, Taiwan \\ 6 Department of Exercise and Health Promotion, Chinese Culture University, Taipei, Taiwan, Taiwan \\ Corresponding Author: Frank Jing-Horng Lu \\ Email address: frankjlu@gmail.com
}

Background: Although Perceived Stress Scale (PSS, Cohen, Kamarack, Mermelstein, 1983) has been validated and widely used in many domains, there is still no validation in sports by comparing athletes and non-athletes and examining related psychometric indices. Purpose: The purpose of this study was to examine the measurement invariance of PSS between athletes and non-athletes, and examine construct validity and reliability in the sports contexts. Methods: Study 1 sampled 359 college student-athletes (males $=233$; females $=126$ ) and 242 non-athletes (males $=124$; females $=118$ ) and examined factorial structure, measurement invariance and internal consistency. Study 2 sampled 196 student-athletes (males $=139$, females $=57$, Mage $=19.88 \mathrm{yrs}, \mathrm{SD}=1.35$ ) and examined discriminant validity and convergent validity of PSS. Study 3 sampled 37 student-athletes to assess test-retest reliability of PSS. Results: Results found that 2-factor PSS-10 fitted the model the best and had appropriate reliability. Also, there was a measurement invariance between athletes and non-athletes; and PSS positively correlated with athletic burnout and life stress but negatively correlated with coping efficacy provided evidence of discriminant validity and convergent validity. Further, the test-retest reliability for PSS subscales was significant ( $r=.66$ and $r=.50)$.

Discussion: It is suggested that 2-factor PSS-10 can be a useful tool in assessing perceived stress either in sports or non-sports settings. We suggest future study may use 2-factor PSS-10 in examining the effects of stress on the athletic injury, burnout, and psychiatry disorders. 
1 Psychometric Properties of the Perceived Stress Scale (PSS): Measurement Invariance Between

2

3

6

$7 \quad{ }^{1}$ National Taiwan Sport University

$8 \quad{ }^{2}$ Chinese Culture University

$9 \quad{ }^{3}$ National Dong Hwa University

$10{ }^{4}$ National Taiwan University of Sport

$11{ }^{5}$ National Chia-Yi University

12

13

14 15

16

17

18

19

20

21

22

23

24

25

26

27

28

29

30

3 Corresponding Author: Frank J.H. Lu

e-mail: frankjlu@gmail.com

Tel: 1-886-933029265

Fax:1-886-22861-7084

9

0

Athletes and Non-athletes and Construct Validity

${ }^{1}$ Yi-Hsiang Chiu, ${ }^{* 2}$ Frank J.H. Lu, ${ }^{3}$ Ju-han Lin, ${ }^{4}$ Chiao-Lin Nien, ${ }^{5}$ Ya-Wen Hsu

$\&{ }^{2}$ Hong-Yu Liu

4 Graduate Institute of Sport Coaching Science, Chinese Culture University

5 \#55, Hwa-Kang Road, Yang-Ming-Shan, Taipei, Taiwan 114 
34 Psychometric Properties of the Perceived Stress Scale (PSS): Measurement Invariance Between Athletes and Non-athletes and Construct Validity

Abstract

Background: Although Perceived Stress Scale (PSS, Cohen, Kamarack, Mermelstein, 1983) has been validated and widely used in many domains, there is still no validation in sports by comparing athletes and non-athletes and examining related psychometric indices. Purpose: The purpose of this study was to examine the measurement invariance of PSS between athletes and non-athletes, and examine construct validity and reliability in the sports contexts. Methods: Study 1 sampled 359 college student-athletes $($ males $=233$; females $=126)$ and 242 non-athletes (males=124; females=118) and examined factorial structure, measurement invariance and internal consistency. Study 2 sampled 196 student-athletes (males $=139$, females $=57, M_{\text {age }}$ $=19.88 \mathrm{yrs}, S D=1.35$ ) and examined discriminant validity and convergent validity of PSS. Study 3 sampled 37 student-athletes to assess test-retest reliability of PSS. Results: Results found that 2-factor PSS-10 fitted the model the best and had appropriate reliability. Also, there was a measurement invariance between athletes and non-athletes; and PSS positively correlated with athletic burnout and life stress but negatively correlated with coping efficacy provided evidence of discriminant validity and convergent validity. Further, the test-retest reliability for PSS subscales was significant $(\mathrm{r}=.66$ and $\mathrm{r}=.50)$.

Discussion: It is suggested that 2-factor PSS-10 can be a useful tool in assessing perceived stress either in sports or non-sports settings. We suggest future study may use 2-factor PSS-10 in examining the effects of stress on the athletic injury, burnout, and psychiatry disorders.

Keywords: cognitive-transactional model of stress, multiple group comparisons, nested model, perceived coping 
61 Since the development of the perceived stress scale (PSS, Cohen, Kamarack, Mermelstein, 62 1983) it has been widely used in various research such as the degree of global stress of a given 63 situation (Leon, Hyre, Ompad, DeSalvo, \& Muntner, 2007; McAlonan et al., 2007), or 64 effectiveness of an intervention on psychological stress (Holzel et al., 2010; Seskevich \& Pieper, 65 2007; Taylor-Piliae, Haskell, Waters, \& Froelicher, 2006), or the associations of perceived stress and psychiatric/physical disorder (Culhane, Rauh, McCollum, Hogan, Agnew, \& Wadhwa, 2001;

67 Garg et al., 2001). In addition, many studies used PSS to examine its relationship with quality of 68 life (Golden-Kreutz, Browne, Frierson, \& Anderson, 2004; Golden-Kreutz et al., 2005), job satisfaction (Norvell, Walden, Gettelman, \& Murrin, 1993), immune functioning (Burns, Drayson, Ring, \& Carroll, 2002; Maes \& Van Bockstaele, 1999), depression (Carpenter et al., 2004), and sleep quality (Cohen \& Williamson, 1988). Therefore, it can be said PSS is a very important tool in assessing stress.

Built on Lazarus and Folkman's (1984) transactional model of stress, the development of PSS is to assess one's perceived nonspecific stress in a given situation or a daily life situation. Lazarus and Folkman's (1984) transactional model of stress contends that an individual's stress perception derived from the imbalance between one's appraisal of situational demands and coping resources. If one perceives that situational demands over resources and the consequences of such failure will be severe; then it will lead to psychophysiological responses such as fast heartbeats, pale face, cold and sweaty hands, tense muscles...etc. Cohen and colleagues (Cohen et al., 1983; Cohen \& William, 1988) constructed this global stress perception by two important components --- something that one can control (i.e., counter stress) and something that one can't control (i.e., perceived stress). In such manner, PSS is not only a measure to assess an extant of how a given situation might hurt oneself but also to assess the degree of how this give situation is controllable or uncontrollable (Golden-Kreutz et al., 2004; Örücü \& Demir, 2009; Roberti, Harrington, \& Storch, 2006).

Given that the applicability of PSS in assessing perceived stress in many domains, the number of the item, the factorial structure, and the reliability of PSS have been intensively examined by many researchers. For example, although the initial version of PSS (Cohen et al., 89 1983) has been developed as 14-item with a unidimensional measure, Cohen and colleagues 90 (Cohen \& William, 1988) continuingly examined the appropriateness of the item's number and 
91 factorial structure of PSS. They sampled 960 male and 1,427 female US residents

92 (Mage $=42.8 \pm 17.2$ years) and examined its factorial structure, criterion validity, and internal

93 consistency of the PSS. Results found 10-item, by deleting item 4, 5, 12, and 13, 2-factor PSS-10

94 can be a better measuring tool of perceived stress because they found the revised version of PSS-

9510 accounted $48.9 \%$ of the variance, and had better reliabilities (Cronbach's $\alpha=.84 \sim .86$ ), and

96 correlated with anxiety, depression and life events which indicated good construct validity.

97 Followed Cohen and colleagues (Cohen \& William, 1988), Hewitt and colleagues (Hewitt,

98 Flett, \& Mosher, 1992) sampled psychiatry patient as participants and examined factorial structure and reliability of PSS, the exploratory factor analysis (EFA) found PSS had two factors --- perceived distress (Cronbach's $\alpha=.81$ ) and perceived coping (Cronbach's $\alpha=.72$ ) with 11 items. Similar findings also found in a Mexico sample where Ramírez and Hernández (2007)

102 found two factors PSS-10 had better reliability and accounted $48.02 \%$ of the variance. Recently, 103 Barbosa-Leiker and colleagues (Barbosa-Leiker, Kostick, McPherson, Roper, Hoekstra, \& 104 Wright, 2013) examined measurement invariance of PSS across gender and time with a clinical sample. Results indicated the 2-factor 10-PSS model provided acceptable fit in both men and women at each time point.

107 Based on above literature, we understand that earlier version of one-factor 14-PSS (Cohen et al., 1983) was designed to measure a global perceived stress without considering the dimensionality. However, when researchers continuingly examined the psychometric properties of PSS (e.g., Barbosa-Leiker, et al., 2013; Cohen \& William, 1988; Hewitt, et al., 1992; Ramírez

111 \& Hernández, 2007) it has found that the items, the factorial structure and the reliability of PSS

112 need further examination. Especially, researchers concerned about whether 2-factor 10-PSS or

113 14-PSS can be an ideal tool in assessing perceived stress. Further, since PSS was to assess

114 individuals perceive normal stress in a given situation or a daily life situation, however, the stress

115 of an athlete on the training and competing situation is different from non-athletes. We doubt

116 whether PSS is suitable to be used in sports and the measurement is invariance comparing to

117 non-athletes. Therefore, it is imperative to examine the psychometric properties of PSS in sports

118 and compare the measurement invariance between athletes and non-athletes.

119 In sports, stress is an important issue that has been received much of attention; specifically 120 in the studies of athletic burnout and athletic injury. For example, Smith (1986) proposed a 
121 cognitive-affective model of athletic burnout to explain the influences of stress on burnout.

122 Smith (1986) explains that in the stressful sports settings, athletes keep appraising contextual

123 stressors and personal coping resources. If athletes perceived situational demands surpass

124 personal resources and consequences will be severe, the cognitive appraisal leads to severe

125 physiological and psychological responses--- anxiety, tension, insomnia, and illness, which

126 eventually lead to burnout. With the same line of conceptualization, Andersen and Williams

127 (1988) also proposed a 'stress-athletic injury model' which contends that athletic injury is the

128 interaction between personality, history of stressors, coping resources and cognitive appraisal. In

129 the stress appraisal process, athletes' perceived stress is influenced by above mentioned factors

130 such as personality, history of stressors, and coping resources. The consequences of this

131 interaction can lead to either attenuate or deteriorate the perceived stress and eventually cause

132 athletic injury.

133 Many researchers borrow either stress-burnout or stress-injury concepts to examine the role

134 of perceived stress on athletes' burnout and injury. Researchers use different measures to do their

135 studies. For example, Rushall (1990) used Daily Analysis of Life Demands for Athletes

136 (DALDA) to measure athletes' perceived environment stimuli and overtraining. Petrie (1992)

137 used the Life Event Scale for Collegiate Athletes (LESCA) to measure athlete's life stressors and

138 its relations to sports injury. Lu and his colleagues (Lu, Lee, Chang, Chou, Hsu, Lin, \& Gill,

139 2016) used College Student-Athlete Life Stress Scale (CSALSS) to examine the relationships

140 between coaches' social support, athletes' resilience and burnout. The general problem of these

141 measures is they are only used for assessing life stressors and examining its' relationship with

142 athlete burnout or sports injury. Although some report adequate psychometric properties (e.g., Lu

143 et al., 2012), but some have been questioned regarding its reliability and validity (e.g., DALDA,

144 LESCA). If they are used for assessing global perceived stress they are not appropriate.

145 To assess global perceived stress in sports, some researchers used PSS in examining its'

146 relationship with sports injury and burnout. For example, in examining whether stress and affect

147 as the mediator of hope-stress relationship, Gustafsson and colleagues (Gustafsson, Skoop,

148 Podlog, Lundqvist, \& Wagnsson, 2013) administered 238 Swedish soccer players with trait hope,

149 Swedish version of PSS (i.e., PSS-10), positive and negative affect and athlete burnout scales.

150 Results found athletes' hope and burnout were fully mediated by stress and positive affect. 
151 Similarly, Tashman,Tenenbaum, and Eklund (2010) sampled 177 college coaches and examined

152 the relationship between coaches' perfectionism and burnout. Results indicated that perceived

153 stress (measured by PSS-14) mediated the relationship between self-evaluative perfectionism and

154 burnout, and a significant direct link to burnout, accounting for $56 \%$ of its variance. Similar sport

155 burnout studies that using either PSS-10 or PSS-14 can be found in Raedeke and Smith (2004),

156 Smith, Gustafsson and Hassmén (2010), and Gustafsson and Skoog (2012) studies.

157 In terms of stress- athletic injury relationship, Galambos and colleagues (Galambos, Terry,

158 Moyle, \& Locke, 2005) investigated 845 Australian youth athletes' injury rates and its relations

159 with psychological variables. Participants (males=433; females=412) completed a demographic

160 questionnaire, health history, Brunel Mood Scale and PSS-10. Results found mood and stress

161 collectively predicted injury characteristics. In a similar study, Malinauskas (2010) sampled 123

162 college athletes and administered with social support scale, PSS-10, and life satisfaction scale.

163 Results found greater perceived stress was associated with diminished life satisfaction for major

164 injury athletes than minor injury athletes. Also, the interaction between perceived stress and

165 perceived social support was associated the most with diminished life satisfaction for athletes

166 with a major injury.

167 As previously mentioned, although sports researchers used either PSS-10 or PSS-14 in

168 examining their relationships with athletic burnout or injury, the psychometric properties of PSS

169 have never been examined in sports. Thus, there are several questions remained. First, whether

170 two-factor or unidimensional PSS will be suitable for sports? Second, if we compare athletes and

171 non-athletes will measurement characteristics remains the same? Third, what is the reliability

172 and validity of PSS in sports? Therefore, the existing knowledge gap of the application of PSS in

173 sports is that we don't know which type of PSS is suitable in terms of dimensionality, construct

174 validity, reliability, and group comparisons between athletes and non-athletes. To fill the gap,

175 there are three purposes in this study. First, we intended to examine the factorial structure of the

176 PSS-10 and PSS-14 and internal consistency. Second, we intended to examine the measurement

177 invariance of PSS between athletes and non-athletes. Third, we attempted to examine the

178 construct validity of PSS and test-retest reliability of PSS.

179

180 Study 1 
181 The purpose of study1 was threefold: (a) to compare the factorial structure of the PSS-10 and

182 PSS-14; (b) to examine internal consistency of PSS-10 and PSS-14; (c) to examine

183 measurement invariance of PSS between athletes and non-athletes.

184 Methods

$185 \quad$ Participants \& procedure

186 We recruited 359 college student-athletes (males $=233$; females $=126)$ with mean age

$18720.08(\mathrm{SD}=+1.51)$ from two sport-colleges and three universities in Taiwan. At the time of data

188 collection, athletic participants were all in their regular training seasons and had been

189 participating in a variety of individual and team sports, such as gymnastics, track and field, golf,

190 weightlifting, basketball, volleyball, soccer, Tae-kwon-do, badminton and baseball for 8.93 years

$191(\mathrm{SD}=3.14)$ of training and competition experiences.

192 For non-athlete participants we recruited $242($ males=124; females=118; Mage $=20.10$

193 yrs, $\mathrm{SD}=+1.55)$ participated in our study. We collected their data during their classes. They are

194 all regular students studied at different academic departments such as accounting, political

195 science, biology, mathematics... etc. They participated in our study voluntarily without any

196 conditional requirements from the classes.

197 After the approval by a local institutional review board (Antai- Tian-Sheng memorial

198 Hospital Institutional Review Board, TSMH IRB No. 15-055-B1), the first author contacted the

$199 \mathrm{coach} /$ teacher of a target team and class and asked permission to use his/her team as participants.

200 Once the coach/teacher agreed to use his/her team as participants, we visited target team/class

201 one hour before they finished the regular training/class. Before administering the questionnaire

202 package, the second author explained the general purpose of the study, the method to complete

203 questionnaires and rights of being a participant. To prevent social desirability effects, we 
204 informed participants that this is a study to explore college students' life experiences, and there

205 were no right or wrong answers. Additionally, we asked them to answer the questions as

206 truthfully as possible, and all responses would be confidential. After the briefing, participants

207 who interested in this study then signed a consent form and completed the demographic

208 questionnaire and 14-item PSS. It took about 15 minutes to complete the questionnaires.

\section{Measurements}

\section{Demographic Information}

211 The demographic questionnaire was designed to gather information about participants' age,

212 gender, types of sports, and years of athletic experiences.

\section{Perceived Stress Scale (PSS)}

214 The PSS is a self-report measure designed to assess one's perception about the degree of a given

215 situation in daily life is considered stressful (Cohen et al., 1983). The PSS-14 contains seven

216 positively worded 'stress' items (e.g., How often have you felt upset because of something that

217 happened unexpectedly?) and seven negatively worded 'counter-stress' items (e.g., How often

218 have you felt confident about your ability to handle personal problems?). Items are rated on a 5-

219 point Likert scale of occurrence these statements over the past 4 weeks $(0=$ never, $1=$ almost

220 never, 2 = sometimes, 3 = fairly often, $4=$ very often). Because reverse-coding may confound

221 the counter-stress factor (Golden-Kreutz et al., 2004), we did not reverse-code the items.

\section{Analytic strategy}

223 All primary statistical testing was conducted in AMOS version 22. Models of the PSS-14 and the 224 PSS-10 (i.e., by deleting item 4, 5, 12, and 13) were first estimated separately for the 1-factor 225 and 2-factor as Table 1. Overall, model fit was evaluated using the following indices suggested 226 by $\mathrm{Hu}$ and Bentler (1999) as follow: the comparative fit index (CFI; study criterion $\geq 0.950$ as 227 ideal and $\geq 0.90$ as the minimum acceptable level), the root mean square error of approximation 228 (RMSEA; study criterion $\leq 0.080$ ) and the standardized root mean square residual (SRMR; study 229 criterion $\leq 0.080$ ). To examine the internal consistency of the factors, Cronbach's $\alpha$ coefficient 230 was used as an index. 
231 For testing measurement invariance, we adopted earlier suggestion (Barbosa-Leiker et al., 232 2011) by following procedures: (a) once the confirmatory factor models for each group

233 established that the overall model was acceptable, a series of analyses to examine measurement

234 invariance were performed sequentially between comparison and nested model; (b) each model

235 was added equality constraints and was tested against the less-constrained model including

236 following indices:

237 1. Configural invariance (Horn \& McArdle, 1992) (also referred to as 'equal form'). This step 238 examined the pattern of salient and non-salient loadings across groups (Vandenberg \& Lance, 239 2000). This step took the measurement model and examined if the theoretical framework of the 240 PSS is the same for athletes and non-athletes.

241 2. Metric invariance (Horn \& McArdle, 1992) (also referred to as 'equal loadings'). This step 242 constrained the factor loadings for like items across groups to determine whether the expected 243 changes in observed values of the indicators per unit change of the construct were equal

244 (Vandenberg \& Lance, 2000). This step tested if the relationships of the PSS-14 or PSS-10 items 245 were equivalent for like indicators in athletes and non-athletes.

246 3. Factor variance/covariance invariance (also referred to as 'equal factor variances'). This step 247 constrained the like factor variances across the groups (Vandenberg \& Lance, 2000). If factor 248 variance invariance holds, then the amount of within group variability of the latent factor is equal 249 across groups (Brown, 2006). This step tested whether athletes and non-athletes use equivalent 250 ranges of the latent constructs (stress and counter-stress) to respond to the PSS-14 or PSS-10 251 items.

252 4. Error variance invariance. This step constrained error variances across the groups. If the same 253 level of measurement error is present for each item between groups. This step tested whether the 254 error of stress and counter-stress all items were related equivalently across athletes and non255 athletes.

256 For tests of invariance, $\chi^{2}$ difference tests are typically used to compare nested models. However, 257 the $\chi^{2}$ difference test may also be influenced by sample size (Chen, Sousa, \& West, 2005); thus, 
258 a change in the comparative fit index (CFI) between comparison and nested models of greater

259 than or equal to -0.010 . In addition, we examined the change in root mean square error of

260 approximation (RMSEA) $\geq 0.015$ or a change in standardized root mean square residual (SRMR)

$261 \geq 0.030$ (for loading invariance) and $\geq 0.010$ (for intercept invariance) is recommended as an

262 appropriate criterion indicating a decrement in fit between models (Chen, 2007; Chen, Sousa, \&

263 West, 2005; Cheung \& Rensvold, 2002). Additionally, a $\chi^{2}$ difference test for a small difference

264 between models (rather than 0) was also conducted ( $\chi^{2}$ critical 0.05; MacCallum, Browne, \& Cai, 265 2006).

\section{Results}

267 Table 1 compares the fit of the 1-factor and 2-factor model of PSS-10 and PSS-14.

268 Both 1-factor model of 14-items $(\mathrm{RMSEA}=0.156>0.080 ; \mathrm{CFI}=0.483<0.90$; $\mathrm{SRMR}=$

$2690.157>0.080)$ and 10 -items $(\mathrm{RMSEA}=0.146>0.080 ; \mathrm{CFI}=0.685<0.90 ; \mathrm{SRMR}=$

$2700.146>0.080)$ did not fit very well. Only 2-factor PSS-10 (RMSEA $=0.070<0.080$; CFI

$271=0.929>0.90 ; \mathrm{SRMR}=0.060<0.080)$ was the best model. Also, it was found Cronbach's

$272 \alpha$ coefficients for 2 -factor PSS-10 were .81 (perceived stress) and .71 (counter stress).

273

274

275

276

277

278

279

280

281

282
(Insert Table 1 about here)

Table 2 shows the athletes and non-athletes 2-factor PSS-10 model of the measurement invariance, M1 was configuration invariance model, M2 metric invariance, M3 variation \ covariance invariance, M4 error variance invariance is shown to have an acceptable adaptation indicators. $\triangle \mathrm{CFI}$ indicated that 2-factor PSS-10 model of athletes and non-athletes in M1, M2, M3 measurement invariance model display equivalent ( $\triangle \mathrm{CFI}<0.01$ ), however, M4 shows the residuals are not equal ( $\triangle \mathrm{CFI}>0.01$ ). We will discuss this later in the discussion.

\section{Study 2}


283 The purpose of study 2 was to examine the construct validity of 2 - factor PSS-10, which is the

284 convergent and discriminant validity, via correlational analyses surrounding the relationships 285 among PSS-stress, PSS counter-stress, college student-athletes' life stress, coping self-efficacy 286 and burnout experiences.

(Insert Table 2 about here)

289

290

291

292

293

294

295

296

297

298

299

300

301

302

303

304

305

\section{Methods}

\section{Participants \& procedure}

A new sample of the targeted population was recruited. Valid data of 196 student-athletes from ten different universities were collected $\left(\right.$ males $=139$, females $=57, M_{\text {age }}=19.88 \mathrm{yrs}, S D=$ 1.35). The recruiting procedure was similar to study 1.

\section{Measurements}

The measurements included the Demographic Questionnaire and the 10-item PSS. In addition, the researchers administered the following measures for examining convergent and discriminant validity.

\section{Athlete Burnout (ABQ)}

ABQ (Raedeke \& Smith, 2001) is a self-reported inventory that assesses athletes' burnout experiences. The initial factor analyses by Raedeke and Smith (2001) revealed that ABQ has three subscales including (a) 5 items for reduced sense of athletic accomplishment, (b) 5 items for perceived emotional and physical exhaustion, and, (c) 5 item for the devaluation of sports participation. Participants identify their athletic burnout experiences using a six-point Likert scale that ranged from 1 (never) to 6 (always). In the present study, the result of CFA confirmed that the factorial structure was suitable for the data. The Cronbach's $\alpha$ for the three subscales 
306 ranged from .63 to .86 and the reliability for all items was .90. To further identify convergent

307 validity it is expected that the burnout scale should be positively correlated with the PSS stress

308 because athletes' stress has been identified as a leading factor of athlete burnout (Lewis, 1991;

309 Nicholls Backhouse, \& McKenna, 2009; Galambo et al., 2005; Johnson \& Ivarsson, 2011). The

310 PSS counter-stress factor was expected to have a negative relation with ABQ.

311 College Student-Athletes’ Life Stress Scale (CSALSS)

312 The 24-item CSALSS (Lu, Hsu, Chan, Cheen, \& Kao, 2012) was used to assess situations

313 that athletes encountered in their daily life and sports and considered as major stressors in their

314 lives. The questionnaire asked questions such as "I am annoyed with my coach's bias against

315 me." There are eight factors in the 24-item CSALSS including: (a) sports injury, (b) performance

316 demand, (c) coach relationships, (d) training adaptation, (e) interpersonal relationships, (f)

317 romantic relationships, (g) family relationships, and (h) academic requirements. Lu and

318 colleagues (Lu et al., 2012) reported that CSALSS can be categorized into two major

319 components --- general life stressors (by adding factor e, f, g, h) and sport-specific stressors (by

320 adding factor a, b, c, d). Participants indicated the frequency of the event on a six-point Likert

321 scale ranged from 1 (Never) to 6 (Always). Cronbach's $\alpha$ of these factors ranged from .69 to .87

322 and the reliability for all items was .92 in this study, indicating that the result was reliable. Given

323 that CSALSS represents an individual's life stress, the PSS counter-stress factor was expected to

324 have negative with CSALSS, while PSS stress was expected to have a positive relation with

325 CSALSS.

326 Coping Self-Efficacy Scale (CSE)

327 CSE (Chesney, Neilands, Chambers, Taylor, \& Folkman, 2006) is a self-reported inventory 328 that assesses one's confidence in performing coping behaviors when faced with life challenges. 
329 The initial factor analyses by Chesney et al. (2006) revealed that CSE has three subscales

330 including (a) problem- focused coping (6 items), (b) stop unpleasant emotions and thoughts (4

331 items), and (c) get support from friends and family (3 items). The CSE uses an eleven-point

332 Likert scale that ranged from 0 ('can not do at all'), 5 ('moderately certain can do') to 10

333 ('certain can do'). In the present study, the Cronbach's $\alpha$ for the three subscales ranged from

334.70 to .78 and the reliability for all items was .86. It is expected that CSE will be negatively

335 correlated with the PSS stress but positively correlated with PSS counter-stress.

\section{Statistical Analyses}

337 To examine the factor structure of the two factors PSS-10, maximum likelihood CFA

338 using AMOS 22.0 was performed, and the researchers reported the following absolute and

339 incremental fit indices if the 2-factors measurement model fit the data well: (1) the $\chi^{2} / \mathrm{DF}$ ratio;

340 (2) the root mean square error of approximation (RMSEA); (3) the standardized root mean

341 square residual (SRMR); (4) the Goodness of Fit Index (GFI); (5) the Comparative Fit Index

342 (CFI); and (6) the Non-Normed Fit Index (NNFI). The recommendations for good fit are the

343 following: $\chi^{2}$ DF ratios between one and three, values for RMSEA values less than 0.08 along

344 with SRMR values less than 0.05 , and GFI/CFI/NNFI values greater than 0.90 (McDonald \& Ho,

345 2002; Hu \& Bentler, 1999). To examine the internal consistency of the factors, Cronbach's $\alpha$

346 coefficient was used as an index. Additionally, the composite reliability (CR $>$.7) (Fornell \&

347 Larcker, 1981) and average variance extracted (AVE > .5) (Kline, 1998) were calculated to

348 examine the fit of internal structure. Finally, using SPSS 18.0, the investigators examined the

349 concurrent and discriminant validity by examining the correlations among PSS, CSALSS, CSE

350 and $\mathrm{ABQ}$. 
351

352

353

354

355

356

357

358

359

360

361

362

363

364

365

366

367

368

369

370

371

372

373

\section{Results}

The results of factorial structure suggest that 2-factor PSS-10 has better measuring quality than unidimensional PSS-10 or PSS-14. The two factors measurement model of the PSS-10 indicated a good fit of the instrument according to the fit indices in study2 (RMSEA $=.056$, $\left.\mathrm{SRMR}=.061, \chi^{2} / \mathrm{DF}=1.607, \mathrm{CFI}=.960, \mathrm{NNFI}=.903, \mathrm{GFI}=.955\right)$. The factor loadings for the 10 items range from .33 to .87 (Figure 1). Cronbach's $\alpha$ coefficients of stress was .77, the coefficient for the "counter-stress" was .68. The composite reliability (Fornell \& Larcker, 1981) for each subscale was calculated: stress (.78), and counter-stress (.73) indicating that each was above the .70 standard. The average variance extracted was also calculated: stress (.59), and counter-stress (.52), which were all above the acceptable standard (.50). As for concurrent and discriminant validity, the Pearson correlations indicated that PSS-stress negatively correlated with CSE total scores and CSE subscales, and positively related to ABQ total scores, ABQ subscales, CASLSS total scores, and CASLSS subscales. Moreover, PSS-counter stress negatively or not correlated with ABQ total scores, ABQ subscales, CASLSS total scores, and CASLSS subscales, in contrast, positively related with CSE (see Table3).

(Insert Table 3 about here)

\section{Study 3}

Previous studies had found that the retest reliability was a better index of the reliability than the internal consistency coefficients (McCrae, 2014; McCrae, Kurtz, Yamagata \& Terracciano, 2011). Therefore, the purpose of study 3 was to examine the test and retest reliability of 2- factor PSS-10.

\section{Methods}

\section{Participants \& procedure}


Another new sample was recruited from Judo and Boxing $(n=39)$. The data was collected

375

376

377

378

379

380

381

382

383

384

385

386

387

388

389

390

391

392

393

394

395

396

397

in 8-9 days, and the general procedures in the previous section. However, due to 2 participants

were unable to complete all items their data were dropped from the study.

\section{Measurements}

The measurements included the Demographic Questionnaire and the 2-factor PSS-10.

\section{Statistical Analyses}

We used SPSS 18.0 to analyze the raw data. The Pearson correlations were used to assess the test-retest reliability.

\section{Results}

Results indicated that the Pearson coefficients for perceived stress $(\mathrm{r}=.66, \mathrm{p}<.00)$, and counter stress was $(r=.50, \mathrm{p}<.00)$ which indicated that two subscales significantly reliable over measuring time. 
398 2-factor PSS-14, or 1-factor PSS-10/PSS-14. The 2-factor PSS-10 reduces 4 items it allows

399 researchers collect data in a short period of time (Shacham, 1983). Although researchers have

400 different arguments regarding dimensionality of PSS (Hewitt, Flett, \& Mosher, 1992; Mitchell,

401 Crane \& Kim, 2008; Örücü, \& Demir, 2009) it is for sure that 2-factor PSS-10 receive better

402 support in sports contexts. The results are consistent with the earlier study by Hewitt and

403 colleagues (Hewitt et al., 1992). Past research in sports generally just assessed life stressors (e.g.,

404 CSALSS, DALDA, LESCA) or arbitrarily used PSS-14/PSS-10 in examining the relationship

405 between stress and related psychological responses (e.g., Raedeke \& Smith,2004; Smith,

406 Gustafsson, and Hassmén,2010; Gustafsson \& Skoog,2012). They did not exactly understand the

407 psychometric properties of PSS. Our results provide robust evidences that 2-factor PSS-10 can

408 be an appropriate tool in the sports settings. Thus, researchers not only have a better tool in

409 examining the relationship between perceived stress on related psychological responses but also

410 practitioners can use 2-factor 10-PSS in evaluating athletes' existing perceived stress. Also, both

411 researchers and practitioner can use two factors--- perceived stress and counter stress to further

412 understand what factor plays an important role on athletes' psychological responses. We suggest

413 future researchers may use 2-factor PSS-10 to examine gender differences, or to compare which

414 variable predict related psychological disorders/constructs the most.

415 In terms of measurement invariance, we found configuration invariance, metric invariance,

416 and variance/covariance invariance are all equivalent except error variance invariance.

417 Therefore, it means that the same level of measurement error for each item between athletes and

418 non-athletes is not the same. However, Lee (2006) suggested that most research that using CFA

419 focus on the equivalence of the factor loadings and factorial covariance. If these indicators meet

420 criteria they can assure that means measurement invariance across observed groups is held, while

421 residual restrain model may be too critical to be reached. Tabachnick and Fidell (2001) also

422 suggest that when factor loadings and factorial covariance are equivalent across group it is

423 indicated that measurement invariance holds true.

424 A significant feature of this study is the sample recruited from Taiwanese student-athletes.

425 Although PSS has been validated in different culture such as Spain (Remor, 2006), Swedish

426 (Eskin, \& Parr, 1996), Japanese (Mimura, \& Griffiths, 2004), Portugal (Ramírez \& Hernández, 
427 2007), Turkish (Örücü, \& Demir, 2009), or Chines (Leung, Lam, \& Chan, 2010), this is the first

428 study using healthy young athletes as participants in examining psychometric properties of PSS 429 and its relationship with athlete burnout, coping self-efficacy and life stress. Our study not only 430 confirms Leung and colleagues' (2010) psychometric properties of the Chinese version of PSS 431 which indicated that 2-factor 14-PSS, 10-PSS, and 4-PSS have better measurement quality but 432 also extends the applicability of PSS into sports contexts. The results of psychometric validation 433 and measurement invariance can be forwarded to those researchers who interested in 434 psychometric properties of PSS. Further, our construct validity analyses found 2-factor PSS-10 435 correlated with athlete burnout and life stress, but negatively correlated with coping self-efficacy, 436 are worthy of forwarding these messages to sports professionals. As we all know the young 437 athletes face many challenges in their life (Lewis, 1991). They engage in heavy and intensive 438 training/competition all year round. The intensive and heavy training bring lots of stress for 439 young athletes (Weinberg \& Gould, 2015). If coaches and sports professionals fail to monitor 440 athletes' training loading and arrange them with appropriate competition plans they may induce 441 lots of stress and cause burnout. Therefore, teaching young athletes effective coping skills (e.g., 442 fostering social support and time management) and teaching psychological skills (e.g., goal443 setting, relaxation, and imagery) are very important because they can help young athletes to cope 444 with stress from training and competitions.

Further, as previously mentioned that most research in sports either using Smith (1986) cognitive-affective model of athletic burnout or Anderson and Williams (1988) stress-athletic injury model to examine the effects of stress on athlete burnout/injury, we suggest future research may extend research beyond this area. Especially, we suggest that future research may examine the antecedents of perceived stress in sports. For example, sports literature suggests that motivational climate created by coaches and team (e.g., ego-involving climate) is one of the major sources of athletes' stress (Hogue, Fry, Fry, \& Pressman, 2013). Researchers may use 2factor PSS-10 in examining how a sports team's motivational climate predicts athletes' perceived stress. In addition, it is well-documented that the leadership style implemented by coaches (e.g., autocratic) may produce stress for athletes (Horn, Bloom, Berglund \& Packard, 2011). We suggest researchers may examine how coaches' leadership and situational conditions (e.g., 456 competition performance) predicting athletes' perceived stress. Further, since the athletic world 
457 is a challenging setting it is found that some athletes become substance abuse and eating disorder

458 to cope stress (Ansel, 2010). We suggest researchers may use 2-factor PSS-10 as a measuring

459 tool in assessing athletes' perceived stress during off- season, pre-season, and after-season in

460 order to understand their stress level and provided with appropriate interventions.

461 As to the application of 2-factor PSS-10 in other domains, we suggest it can be a useful tool

462 in assessing perceived stress in an intervention study. For example, if researchers want to know

463 whether a stress management program reducing participants' perceived stress (e.g., Shapiro,

464 Astin, Bishop \& Cordova, 2005). In such condition, researchers can use 2-factor PSS-10 before

465 and after intervention so to understand the effectiveness of the intervention. Also, many

466 researchers adopt a psychophysiological approach to examined participants'

467 electroencephalography (EEG) during stressful conditions (e.g., Cavanagh \& Shackman, 2015;

468 Grupe \& Nitschke, 2013; Proudfit, Inzlicht, \& Mennin, 2013). In such case, if researchers use 2-

469 factor PSS-10 combined with EEG it can be a sound approach to detect participants' real

470 psychological reactions. Further, many researchers in medicine intend to examine whether there

471 is an association between perceived stress and coping strategies (e.g., Najam \& Aslam, 2010).

472 For this type of study 2-factor, PSS-10 can be a quick and efficient tool for collecting data.

473 There are several limitations that need to be addressed. First, our sample is all recruited

474 from Division I college student-athletes whether our results could be generalized to other

475 athletes, such as professional athletes or junior athletes, need to be further examined.

476 Additionally, the data was collected from Taiwanese student-athletes; hence the results may not

477 be generalizable to different cultures. We recommend researchers adopt similar approaches to

478 test measurement invariance in different culture and populations.

479

480

481

482

483

484

\section{Conclusion}

To acknowledge that PSS is a widely used measure in assessing stress, we have conducted three studies to examine the factor structure, measurement invariance between athletes and nonathletes, internal reliability, test-retest reliability and construct validity in the sports contexts. Results indicated that 2-factor PSS-10 can be an ideal measure for the research in sports. We suggest future research may use 2-factor PSS-10 in conducting various stress research. 
Andersen, M. B., \& Williams, J. M. (1988). A model of stress and athletic injury: Prediction and prevention. Journal of Sport and Exercise Psychology, 10, 294-306.

Ansel, M. (2010). Drugs in sports: causes and cures. In J. Williams (Ed.). Applied Sport Psychology: Personal Growth to Peak Performance (pp.463-491). New York: McGrawHill.

Barbosa-Leiker, C., Wright, B. R., Burns, G. L., Parks, C. D., \& Strand, P. (2011). Is the

493

494

495

496

497

498

499

500

501

502

503

504

505

506

507

508

509

510

511

512

513 assessment of the metabolic syndrome stable over time? Testing longitudinal measurement invariance of the metabolic syndrome. Annals of Epidemiology, 21, 111-117.

Barbosa-Leiker, C., Kostick, M., McPherson, S., Roper, V., Hoekstra, T., \& Wright, B. (2013). Measurement invariance of the perceived stress scale and latent mean differences across gender and time. Stress and Health, 29, 253-260.

Brown, T. A. (2006). Confirmatory factor analysis for applied research. New York: Guilford Press.

Burns, V. E., Drayson, M., Ring, C., \& Carroll, D. (2002). Perceived stress and psychological well-being are associated with antibody status after meningitis $\mathrm{C}$ conjugate vaccination. Psychosomatic Medicine, 64: 6 , 963-970. doi:10.1097/01.PSY.0000038936.67401.28. PMID 12461201.

Carpenter, L. L., Tyrka, A. R., McDougle, C. J., Malison, R. T., Owens, M. J., Nemeroff, C. B., \& Price, L. H. (2004). Cerebrospinal fluid corticotropin-releasing factor and perceived early life stress in depressed patients and healthy control subjects.

Neuropsychopharmacology, 29: 4, 777-784. doi:10.1038/sj.npp.1300375. PMID 14702025.

Cavanagh, J. F., \& Shackman, A. J. (2015). Frontal midline theta reflects anxiety and cognitive control: Meta-analytic evidence. Journal of Physiology, 109:3, 1-13.

Chesney, M. A., Neilands, T. B., Chambers, D. B., Taylor, J. M., \& Folkman S. (2006). A validity and reliability study of the Coping Self-Efficacy Scale. British Journal of Health Psychology, 11, 421-437. 
514 Chen, F. F. (2007). Sensitivity of goodness of fit indexes to lack of measurement invariance.

515 Structural Equation Modeling, 14, 464-504.

516 Chen, F. F., Sousa, K. H., \& West, S. G. (2005). Testing measurement invariance of second-

517 order factor models. Structural Equation Modeling, 12, 471-492.

518 Cheung, G. W., \& Rensvold, R. B. (2002). Evaluating goodness-of-fit indexes for testing

519 measurement invariance. Structural Equation Modeling, 9, 233-255.

520 Cohen, S., Kamarack, T., \& Mermelstein, R. (1983). A global measure of perceived stress.

$521 \quad$ Journal of Health and Social Behavior, 24, 385-396.

522 Cohen, S., \& Williamson, G. M. (1988). Perceived stress in a probability sample in the United

523 States. In S. Spacapan \& S. Oskamp (Eds.), The social psychology of health (pp. 31-67).

$524 \quad$ Newbury Park, CA: Oxford.

525 Culhane, J. F., Rauh, V., McCollum, K. F., Hogan, V. K., Agnew, K., \& Wadhwa, P. D. (2001).

526 Maternal stress is associated with bacterial vaginosis in human pregnancy. Maternal and

527 Child Health Journal, 5:2, 127-134. doi: 10.1023/A:1011305300690. PMID 11573838.

528 Eskin, M., \& Parr, D. (1996). Introducing a Swedish version of an instrument measuring mental

529 stress. Reports from the Department of Psychology, U Stockholm, 813, 1-9.

530 Galambos, S. A., Terry, P. C., Moyle, G. M. \& Locke, S. A. (2005). Psychological predictors of

531 injury among elite athletes. British Journal of Sports Medicine, 39: 6, 351-354.

532 Garg, A., Chren, M. M., Sands, L. P., Matsui, M. S., Marenus, K. D., Feingold, K. R., \& Elias, P. M. (2001). Psychological stress perturbs epidermal permeability barrier homeostasis: implications for the pathogenesis of stress-associated skin disorders. Archives of Dermatology, 137:1, 53-59. doi: 10.1001/archderm.137.1.53. PMID 11176661

Golden-Kreutz, D. M., Browne, M. W., Frierson, G. M., \& Anderson, B. L. (2004). Assessing stress in cancer patients: a second-order factor analysis model for the Perceived Stress Scale. Assessment, 11, 216-222.

Golden-Kreutz, D. M., Thornton, L. M., Wells-Di, G. S., Frierson, G. M., Jim, H. S., \& Carpenter, K. M. (2005). Traumatic stress, perceived global stress, and life events: prospectively predicting the quality of life in breast cancer patients. Health Psychology, 24, 288-296. 
543

544

545

546

547

548

549

550

551

552

553

554

555

556

557

558

559

560

561

562

563

564

565

566

567

568

569

570

571

572

Gustafsson, H., Skoog, T., Podlog, L., Lundqvist, C., \& Wagnsson, S. (2013). Hope and athlete burnout: Stress and affect as mediators. Psychology of Sport and Exercise, 14, 640649. doi.org/10.1016/j.psychsport.2013.03.008

Grupe, D. W., \& Nitschke, J. B. (2013). Uncertainty and anticipation in anxiety: An integrated neurobiological and psychological perspective. Nature Reviews Neuroscience, 14:7, 488501.

Gustafsson, H., \& Skoog, T. (2012). The mediational role of perceived stress in the relation between optimism and burnout in competitive athletes. Anxiety, Stress, \& Coping. 25, 183 199. DOI:10.1080/10615806.2011.594045

Hewitt, P. L., Flett, G. L., \& Mosher, S. W. (1992). The perceived stress scale: Factor structure and relation to depression symptoms in a psychiatric sample. Journal of Psychopathology and Behavioral Assessment, 14: 3, 247-257.

Hogue, C. M., Fry, M. D., Fry, A. C., \& Pressman, S. D. (2013). The influence of a motivational climate intervention on participants' salivary cortisol and psychological responses. Journal of Sport \& Exercise Psychology, 35, 85-97.

Holzel, B. K., Carmody, J., Evans, K. C., Hoge, E. A., Dusek, J. A., Morgan, L., Pitman, R. K., \& Lazar, S. W. (2010). Stress reduction correlates with structural changes in the amygdala. Social Cognitive \& Affective Neuroscience, 5:1, 11-17. doi:10.1093/scan/nsp034. PMC 2840837. PMID 19776221.

Horn, J. L., \& McArdle, J. J. (1992). A practical and theoretical guide to measurement invariance in aging. Experimental Aging Research, 18, 117-144.

Horn, T. S., Bloom, P., Berglund, K. M., Packard, S. (2011). Relationship between collegiate athletes' psychological characteristics and their preferences for different types of coaching behavior. Sport Psychology, 25: 2, 190-211.

Hu, L., \& Bentler, P. M. (1999). Cut-off criteria for fit indexes in covariance structure analysis: Conventional criteria versus new alternatives. Structural Equation Modeling, 6, 1-55.

Lazarus, R. S., \& Flokman, S. (1984). Stress, appraisal, and coping. New York: Springer.

Lee, M.N. (2006). Structural Equation Modelling: Introduction and Applications of Amos in Measurement Development_ Graphics \& Basic. Taipei: Psychology Publishers [in Chinese]. 
573 Leon, K. A., Hyre, A. D., Ompad, D., DeSalvo, K. B., \& Muntner, P. (2007). Perceived stress

574

575

576

577

578

579

580

581

582

583

584

585

586

587

588

589

590

591

592

593

594

595

596

597

598

599

600

601 among a workforce 6 months following hurricane Katrina. Social Psychiatry and Psychiatric Epidemiology, 42:12, 1005-1011. doi:10.1007/s00127-007-02606. PMID 17932611.

Leung, D. Y. P., Lam, T. H., \& Chan, S. S. (2010). Three versions of Perceived Stress Scale: Validation in a sample of Chinese cardiac patients who smoke, BMC Public Health, 10:513, DOI: $10.1186 / 1471-2458-10-513$

Lewis, M. (1991). Athletes in college: Differencing role and conflicting expectations. College Student Journal , 10, 195-200.

Lu, F. J. H., Hsu, Y. W., Chan, Y. S., Cheen, J. R., \& Kao, K. T. (2012). Assessing college student-athletes' life stress: Initial measurement development and validation. Measurement in Physical Education and Exercise Science, 16:4, 254-267.

Lu, F.J.H., Lee, W.P., Chang, Y.K., Chou, C.C., Hsu, Y.W., Lin, J.H., \& Gill, D.L. (2016). Interaction of athletes' resilience and coaches' social support on the stress-burnout relationship: A conjunctive moderation perspective, Psychology of Sport and Exercise 22, 202-209.

Mahon, N. E., Yarcheski, A., Yarcheski, T. J., \& Hanks, M. M. (2007). Relations of low frustration tolerance beliefs with stress, depression, and anxiety in young adolescents. Psychological Reports, 100, 98-100.

Malinauskas, R. (2010). The associations among social support, stress, and life satisfaction as perceived by injured college athletes. Social Behavior and Personality, 38: 6, 741-752.

Maes, M., \& Van Bockstaele, D. R. (1999). The effects of psychological stress on leukocyte subset distribution in humans: evidence of immune activation. Neuropsychobiology, 39:1, 1-9. doi:10.1159/000026552. PMID 9892853.

MacCallum, R. C., Browne, M. W., \& Cai, L. (2006). Testing differences between nested covariance structure models: power analysis and the null hypothesis. Psychological Methods, 11, 19-35.

McAlonan, G. M., Lee, A. M., Cheung, V., Cheung, C., Tsang, K. W. T., Sham, P. C., Chua, S. E., \& Josephine, G. W. S. (2007). Immediate and sustained psychological impact of an 
602

603

604

605

606

607

608

609

610

611

612

613

614

615

616

617

618

619

620

621

622

623

624

625

626

627

628

629

emerging infectious disease outbreak on health care workers. The Canadian Journal of Psychiatry, 52: 4, 241-247.PMID 17500305

McCrae, R. R. (2014). A more nuanced view of reliability: specificity in the trait hierarchy. Personality and Social Psychology Review, 1-16. doi:10.1177/1088868314541857.

McCrae, R. R., Kurtz, J. E., Yamagata, S., \& Terracciano, A. (2011). Internal consistency, retest reliability, and their implications for personality scale validity. Personality and Social Psychology Review, 15:1, 28-50. doi:10.1177/1088868310366253

McDonald, R. P., \& Ho, M. R. (2002). Principles and practice in reporting structural equation analyses. Psychological Methods, 7, 64-82.

Mimura, C., \& Griffiths, P. (2004). A Japanese version of the perceived stress scale: translation and preliminary test. International Journal of Nursing Study, 41, 379-385. 10.1016/j.ijnurstu.2003.10.009

Mitchell, A.M., Crane, P.A., \& Kim, Y. (2008). Perceived Stress in Survivors of Suicide: Psychometric Properties of the Perceived Stress Scale. Research in Nursing \& Health, 31, 576-585.

Najam, N. \& Aslam, S. (2010). Perceived stress and coping strategies in headache (migraine \& tension-type headache) patients. Journal of Behavioural Sciences, 20, 1-15.

Nicholls, A. R., Backhouse, S. H., Polman, R. C. J., \& McKenna, J. (2009). Stressors and affective states among professional rugby union players. Scandinavian Journal of Medicine and Science in Sports, 19, 121-128.

Norvell, N., Walden, K., Gettelman, T., \& Murrin, M. (1993). Understanding occupational stress in child welfare supervisors. Journal of Applied Social Psychology, 23, 2043-2054.

Nikolaos, A. (2012). An examination of a burnout model in basketball coaches. Journal of Physical Education and Sport, 12: 2, 171 - 179.

Örücü, M. Ç., \& Demir, A. (2009). Psychometric evaluation of Perceived Stress Scale for Turkish university students. Stress and Health, 25, 103-109.

Petrie, T. A. (1992). Psychosocial antecedents of athletic injury: The effects of life stress and social support on female collegiate gymnasts. Behavioral Medicine, 18, 127-138. 
630 Pizzagalli, D. A., Bogdan, R., Ratner, K. G., \& Jahn, A. L. (2007). Increased perceived stress is 631 associated with blunted hedonic capacity: Potential implications for depression research. 632 Behaviour Research and Therapy, 45, 2742-2753.

633 Pollard, L. J., \& Bates, L. W. (2004). Religion and perceived stress among undergraduates 634 during Fall 2001 final examinations. Psychological Reports, 95, 999-1007.

635 Proudfit, G. H., Inzlicht, M., \& Mennin, D. S. (2013). Anxiety and error monitoring: The 636 importance of motivation and emotion. Frontiers in Human Neuroscience, 7, 636.

637 Raedeke, T. D., \& Smith, A. L. (2004). Coping resources and athlete burnout: An examination of 638 stress-mediated and moderation hypotheses. Journal of Sport and Exercise Psychology, 26, $639 \quad 525-541$.

640 Ramírez, M. T. G., \& Hernández, R. L. (2007). Factor structure of the perceived stress scale 641 (PSS) in a sample from Mexico. The Spanish Journal of Psychology, 10: 1, 199-206. 642 Remor, E. (2006). Psychometric properties of a European Spanish version of the perceived stress 643 scale(PSS). The Spanish Journal of Psychology, 9:1, 86-93.

644 Roberti, J. W., Harrington, L. N., \& Storch, E. A. (2006). Further psychometric support for the 645 10-item version of the Perceived Stress Scale. Journal of College Counseling, 9, 135-147.

Rushall, B. R. (1990). A tool for measuring stress tolerance in elite athletes. Journal of Applied Sport Psychology, 2, 51-66.

648 Seskevich, J. E., \& Pieper, C. F. (2007). Brief meditation training can improve perceived stress and negative mood. Alternative Therapies in Health and Medicine, 13:1, 38-44. PMID 17283740.

Shacham, S. (1983). A shorten version of the profile of mood states. Journal of Personality

653 Shapiro, S.L., Astin, J.A., Bishop, S.R., \& Cordova, M. (2005). Mindfulness-based stress 654 reduction for health care professionals: Results from a randomized trial. International 655 Journal of Stress Management, 12:2, 164-176.

656 Smith, R. E. (1986). Toward a cognitive-affective model of athletic burnout. Journal of Sport 657 Psychology, 8, 36-50. 
658 Smith, A. L., Gustafsson, H., \& Hassmén, P. (2010). Peer motivational climate and burnout 659 perceptions of adolescent athletes, Psychology of Sport and Exercise, 11, 453-460.

660 doi:10.1016/j.psychsport.2010.05.007

661 Tabachnick, B. G., \& Fidell, L. S. F. (2001). Using multivariate statistics (4th ed.). Boston: $662 \quad$ Allyn \& Bacon.

663 Tashman, L. S., Tenenbaum, G., \& Eklund, R. (2010). The effect of perceived stress on the 664 relationship between perfectionism and burnout in coaches. Anxiety, Stress, and Coping, $66523: 2,195-212$.

666 Taylor-Piliae, R.E., Haskell, W.L., Waters, C.M., \& Froelicher, E.S. (2006). Change in perceived 667 psychosocial status following a 12-week Tai Chi exercise program. Journal of Advanced $668 \quad$ Nursing, 54: 3, 313-329.

669 Vandenberg, R. J., \& Lance, C. E. (2000). A review and synthesis of the measurement invariance 670 literature: suggestions, practices, and recommendations for organizational research.

671 Organizational Research Methods, 3, 4-69.

672 Weinberg, R., \& Gould, D. (2015). Foundations of sport and exercise psychology (6th ed.).

673 Champaign, IL: Human Kinetics. 
Table $\mathbf{1}$ (on next page)

Fit indices for one-factor and two-factor models of the PSS 


\section{Table 1:}

2 Fit indices for one-factor and two-factor models of the PSS.

\begin{tabular}{cccccc}
\hline & $d f$ & $\chi^{2}$ & CFI & RMSEA & SRMR \\
\hline 1-factor & & & & & \\
PSS14 & 77 & $1171.113^{* *}$ & 0.495 & 0.154 & 0.162 \\
PSS10 & 35 & $486.814^{* *}$ & 0.693 & 0.147 & 0.126 \\
2-factor & & & & & \\
PSS14 & 76 & $361.388^{* *}$ & 0.868 & 0.079 & 0.078 \\
PSS10 & 34 & $100.712^{* *}$ & 0.955 & 0.057 & 0.049 \\
\hline
\end{tabular}

$p<.001^{* *}$

3 


\section{Table 2 (on next page)}

Table.doc

this table illustrate the invariance model of 2-factor PSS 10 
1 Table2: Invariance models of the two-factor PSS-10 between athletes and non-athletes.

\begin{tabular}{|c|c|c|c|c|c|c|c|}
\hline & & $d f$ & $\chi^{2}$ & CFI & RMSEA & SRMR & $\Delta \mathrm{CFI}$ \\
\hline & M1(configural) & 68 & $178.961 * *$ & 0.927 & 0.052 & 0.059 & \\
\hline athlete & M2(metric) & 76 & $193.196^{* *}$ & 0.923 & 0.051 & 0.061 & -0.004 \\
\hline v.s. & M3(variancel & 79 & $196.174 * *$ & 0.923 & 0.050 & 0.062 & 0.000 \\
\hline \multirow[t]{2}{*}{ non-athlete } & covariance) & & & & & & \\
\hline & M4(residual) & 89 & $265.011 * *$ & 0.884 & 0.057 & 0.061 & -0.039 \\
\hline
\end{tabular}

2 


\section{Table 3 (on next page)}

Table3 bi-variate correlations of all variables

this table illustrates all correlations among variables 
1 Table 3:

2 The bivariate correlations of PSS, ABQ, CSE, and CSALSS.

\begin{tabular}{|c|c|c|c|c|c|c|c|c|c|c|c|c|c|c|c|c|c|c|c|}
\hline & 1 & 2 & 3 & 4 & 5 & 6 & 7 & 8 & 9 & 10 & 11 & 12 & 13 & 14 & 15 & 16 & 17 & 18 & 19 \\
\hline 1.Stress & .77 & & & & & & & & & & & & & & & & & & \\
\hline $\begin{array}{l}\text { 2.Counter- } \\
\text { stress }\end{array}$ & -.13 & .68 & & & & & & & & & & & & & & & & & \\
\hline 3.ABQ & $.37^{*}$ & $-.29 *$ & .90 & & & & & & & & & & & & & & & & \\
\hline 4.CSE & $-.29 *$ & $.41^{*}$ & $-.30 *$ & .86 & & & & & & & & & & & & & & & \\
\hline 5.CASLSS & $.45^{*}$ & $-.18^{*}$ & $.46^{*}$ & $-.29 *$ & .92 & & & & & & & & & & & & & & \\
\hline 6.ABQ-RA & $.31^{*}$ & $-.44 *$ & $.82^{*}$ & $-.31^{*}$ & $.36^{*}$ & .63 & & & & & & & & & & & & & \\
\hline 7.ABQ-E & $.34^{*}$ & $-.17^{*}$ & $.86^{*}$ & $-.26^{*}$ & $.39 *$ & $.54^{*}$ & .85 & & & & & & & & & & & & \\
\hline 8.ABQ-D & $.31^{*}$ & $-.18 *$ & $.90^{*}$ & $-.23^{*}$ & $.44^{*}$ & $.65^{*}$ & $.64^{*}$ & .86 & & & & & & & & & & & \\
\hline 9.CSE-PC & $-.15^{*}$ & $.27^{*}$ & $-.19 *$ & $.74^{*}$ & $-.19 *$ & $-.28^{*}$ & -.11 & -.14 & .76 & & & & & & & & & & \\
\hline 10.CSE-SET & $-.32 *$ & $.44^{*}$ & $-.29 *$ & $.92^{*}$ & $-.31^{*}$ & $-.32 *$ & $-.25 *$ & $-.21^{*}$ & $.52^{*}$ & .78 & & & & & & & & & \\
\hline 11.CSE-GF & $-.20 *$ & $.28^{*}$ & $-.26^{*}$ & $.85^{*}$ & $-.22 *$ & $-.17^{*}$ & $-.26 *$ & $-.22 *$ & $.47^{*}$ & $.69 *$ & .70 & & & & & & & & \\
\hline 12.CASLSS-SJ & $.31^{*}$ & $-.24^{*}$ & $.26^{*}$ & $-.24^{*}$ & $.68^{*}$ & $.21^{*}$ & $.27^{*}$ & $.20 *$ & $-.16^{*}$ & $-.23^{*}$ & $-.19 *$ & .87 & & & & & & & \\
\hline 13.CSALSS-PD & $.46^{*}$ & $-.24^{*}$ & $.30 *$ & $-.34^{*}$ & $.65^{*}$ & $.32 *$ & $.28^{*}$ & $.21^{*}$ & $-.19 *$ & $-.37^{*}$ & $-.25^{*}$ & $.56^{*}$ & .69 & & & & & & \\
\hline 14.CSALSS-CR & $.37^{*}$ & -.08 & $.46^{*}$ & $-.21^{*}$ & $.70^{*}$ & $.39 *$ & $.32^{*}$ & $.47^{*}$ & -.13 & $-.21^{*}$ & $-.19 *$ & $.38^{*}$ & $.39 *$ & .84 & & & & & \\
\hline 15.CSALSS-TA & $.40 *$ & $-.15 *$ & $.57^{*}$ & $-.26^{*}$ & $.80 *$ & $.39 *$ & $.50 *$ & $.55^{*}$ & -.10 & $-.30 *$ & $-.23^{*}$ & $.45^{*}$ & $.45^{*}$ & $.64^{*}$ & .70 & & & & \\
\hline 16.CSALSS-IR & $.17^{*}$ & -.06 & $.37^{*}$ & $-.16^{*}$ & $.69 *$ & $.28^{*}$ & $.25^{*}$ & $.42^{*}$ & $-.15^{*}$ & $-.14^{*}$ & -.12 & $.24^{*}$ & $.24^{*}$ & $.51^{*}$ & $.58 *$ & .81 & & & \\
\hline 17.CSALSS-RR & $.19 *$ & -.03 & $.20 *$ & -.05 & $.70^{*}$ & $.14^{*}$ & $.17^{*}$ & $.20 *$ & -.03 & -.09 & .01 & $.31^{*}$ & $.24^{*}$ & $.31^{*}$ & $.52 *$ & $.52^{*}$ & .75 & & \\
\hline 18.CSALSS-FR & $.28 *$ & -.08 & $.23^{*}$ & -.12 & $.79 *$ & $.20 *$ & $.15^{*}$ & $.24^{*}$ & -.13 & -.13 & -.06 & $.42 *$ & $.39 *$ & $.44^{*}$ & $.53^{*}$ & $.60 *$ & $.61^{*}$ & .70 & \\
\hline 19.CSALSS-AR & $.41^{*}$ & $-.17 *$ & $.28^{*}$ & $-.29 *$ & $.74^{*}$ & $.18^{*}$ & $.28^{*}$ & $.25^{*}$ & $-.18^{*}$ & $-.32 *$ & $-.21^{*}$ & $.45^{*}$ & $.50 *$ & $.35^{*}$ & $.48^{*}$ & $.37^{*}$ & $.49 *$ & $.56^{*}$ & .77 \\
\hline Mean & 2.01 & 2.03 & 3.03 & 6.65 & 2.82 & 3.14 & 3.22 & 2.73 & 6.83 & 6.38 & 6.84 & 3.45 & 3.51 & 2.65 & 2.73 & 2.08 & 2.38 & 2.61 & 3.18 \\
\hline$S D$ & 0.67 & 0.70 & 0.85 & 1.37 & 0.79 & 0.80 & 1.03 & 1.13 & 1.88 & 1.61 & 1.46 & 1.29 & 0.94 & 1.19 & 1.00 & 0.97 & 1.15 & 1.08 & 1.21 \\
\hline
\end{tabular}

Note:

$* p<.05$; Cronbach alphas are presented on the diagonal as bold font.

$A B Q-R A=$ reduced sense of athletic accomplishment; $A B Q-E=$ perceived emotional and physical exhaustion; $A B Q-D=$ devaluation of $s p o r t s$ participation. $C S E-P C=$ problem-focused

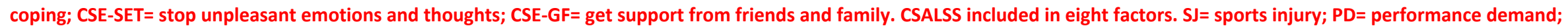
$C R=$ coach relationships; $T A=$ training adaption; $I R=$ interpersonal relationships; $R R=$ romantic relationships; $F R=$ family relationships; $A R=$ academic requirements. 


\section{Table 4 (on next page)}

The two factors measurement model of the PSS-10 


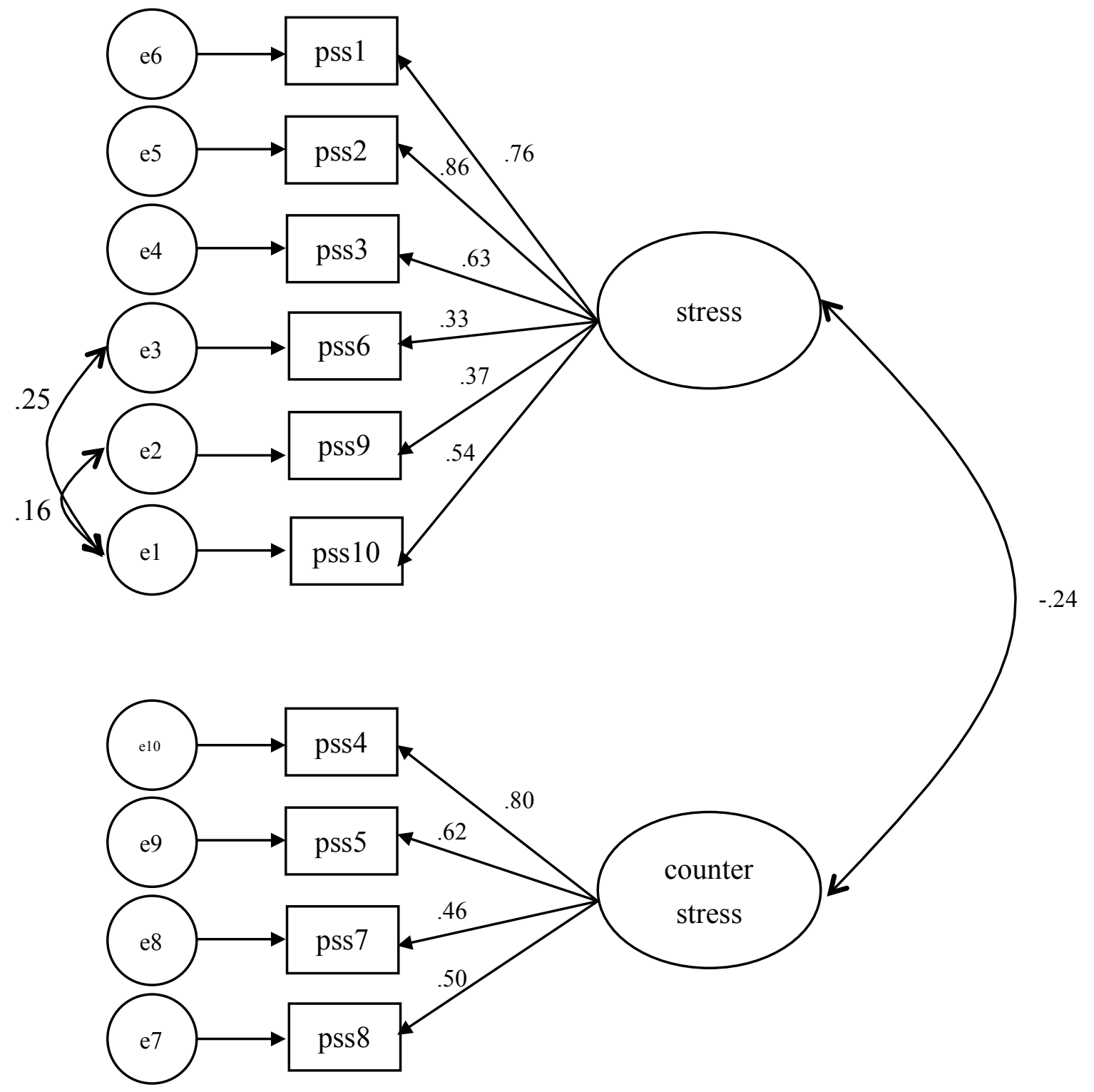

Figure 1 The two factors measurement model of the PSS-1 
